\title{
Wave number selection in the presence of noise: Experimental results
}

Cite as: Chaos 28, 053110 (2018); https://doi.org/10.1063/1.5011349

Submitted: 31 October 2017 . Accepted: 02 May 2018 . Published Online: 22 May 2018

Dmitry Zhilenko (D), Olga Krivonosova, Maria Gritsevich (D), and Peter Read
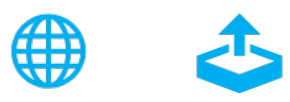

\section{ARTICLES YOU MAY BE INTERESTED IN}

Amplitude mediated chimera states with active and inactive oscillators

Chaos: An Interdisciplinary Journal of Nonlinear Science 28, 053109 (2018); https:// doi.org/10.1063/1.5031804

Understanding transient uncoupling induced synchronization through modified dynamic coupling

Chaos: An Interdisciplinary Journal of Nonlinear Science 28, 053112 (2018); https:// doi.org/10.1063/1.5016148

Relativistic quantum chaos-An emergent interdisciplinary field

Chaos: An Interdisciplinary Journal of Nonlinear Science 28, 052101 (2018); https:// doi.org/10.1063/1.5026904

\section{AlP Author Services English Language Editing}




\title{
Wave number selection in the presence of noise: Experimental results
}

\author{
Dmitry Zhilenko, ${ }^{1}$ Olga Krivonosova, ${ }^{1}$ Maria Gritsevich, ${ }^{2,3, a)}$ and Peter Read ${ }^{4}$ \\ ${ }^{1}$ Institute of Mechanics, Lomonosov Moscow State University, Michyrinskiy Prospect 1, 119192 Moscow, \\ Russia \\ ${ }^{2}$ Department of Physics, University of Helsinki, Gustaf Hällströmin katu 2a, P.O. Box 64, FI-00014 Helsinki, \\ Finland \\ ${ }^{3}$ Department of Physical Methods and Devices for Quality Control, Institute of Physics and Technology, \\ Ural Federal University, Mira Street 19, 620002 Ekaterinburg, Russia \\ ${ }^{4}$ Department of Physics, Atmospheric, Oceanic and Planetary Physics, University of Oxford, Parks Road, \\ Oxford OX1 3PU, United Kingdom
}

(Received 31 October 2017; accepted 2 May 2018; published online 22 May 2018)

\begin{abstract}
In this study, we consider how the wave number selection in spherical Couette flow, in the transition to azimuthal waves after the first instability, occurs in the presence of noise. The outer sphere was held stationary, while the inner sphere rotational speed was increased linearly from a subcritical flow to a supercritical one. In a supercritical flow, one of two possible flow states, each with different azimuthal wave numbers, can appear depending upon the initial and final Reynolds numbers and the acceleration value. Noise perturbations were added by introducing small disturbances into the rotational speed signal. With an increasing noise amplitude, a change in the dominant wave number from $m$ to $m \pm 1$ was found to occur at the same initial and final Reynolds numbers and acceleration values. The flow velocity measurements were conducted by using laser Doppler anemometry. Using these results, the role of noise as well as the behaviour of the amplitudes of the competing modes in their stages of damping and growth were determined. Published by AIP Publishing. https://doi.org/10.1063/1.5011349
\end{abstract}

It is well known for many dynamical systems that both noise and forcing can cause instabilities, shifting of bifurcation parameters, or pattern selection in the flow after the instability. In nature, in spherical flows with rotation, such as flows in the Earth's mantle and the atmosphere, noise and forcing are combined. These flows undergo the action of non-uniform in time Earth rotation and time dependent external heating. To estimate quantitatively the role of noise in the presence of forcing, we carried out experiments on wave number selection for rotating spherical flow, when its state changes from subcritical to a supercritical one. Noise is injected to the flow by relatively small, irregular in time perturbations in the imposed rotation rate and forcing-by rotation with acceleration. The obtained results provide insights into nonlinear interactions and energy exchange between the competing modes in model flows and can be extended to other dynamical systems.

\section{INTRODUCTION}

The occurrence of multiple, coexisting intransitive solutions, either as steady equilibria or more complex, timedependent states in the same region of parameter space, is commonly encountered in strongly nonlinear dynamical systems, especially those possessing certain spatio-temporal symmetries. Numerous examples have been reported among experiments in fluid flow in which the principal mode of

\footnotetext{
a)Author to whom correspondence should be addressed: maria.gritsevich@ helsinki.fi
}

instability is in the form of spatially periodic cells or travelling waves. ${ }^{1-5}$ After the first instability in Taylor-Couette flow, for example, multiple stable solutions with different axial wave numbers can be observed at the same Reynolds numbers, the state observed depending upon the initial flow state and the rate of angular acceleration of one of the rotating boundaries; ${ }^{1,2}$ the accelerations are determined by the initial and final Reynolds numbers and the time of transition between them.

The selection of one from at least two competing linear modes in spherical Couette flow (SCF) - the flow of viscous fluid in the gap between concentric spherical boundaries, induced by their rotation about a common axis-is under study in this paper. Subcritical SCF is stationary and symmetric about the axis of rotation and equatorial plane and loses its stability with increasing Reynolds number: $\operatorname{Re}=\Omega$ $\mathrm{r}_{1}{ }^{2} / \nu$, where $\mathrm{r}_{1}$ is the radius of the inner sphere, $\Omega$ is the rotational angular velocity, and $\nu$ is the kinematic viscosity. Flow structures caused by the first instability are determined by the relative gap size or aspect ratio, $\delta=\left(\mathrm{r}_{2}-\mathrm{r}_{1}\right) / \mathrm{r}_{1}$, where $\mathrm{r}_{2}$ is the radius of the outer sphere. In shallow layers (for which the aspect ratio $\delta<0.24$ ), Taylor vortices ${ }^{6}$ first appear as a result of flow instability; vortices are steady for $\delta>0.02{ }^{7}$ Taylor vortices can be symmetric about the axis of rotation, as well as asymmetric about the equatorial plane. ${ }^{8,9}$ Asymmetric modes can occur in both hemispheres and their location typically depends on random effects. ${ }^{8}$ In wide gaps $(\delta>0.42)$, secondary flow after the first instability takes the form of azimuthal waves, which are generally asymmetric about the equatorial plane and propagate at a rate dependent upon the inner sphere rotation, and whose 
dominant wave number decreases with increasing gap size. ${ }^{10-12}$

The possibility of multiple stable flow states in SCF was found for the first time in shallow layers. ${ }^{13}$ It was demonstrated experimentally ${ }^{14}$ that secondary flows in thin layers with 0,1 , and 2 Taylor vortices can appear in each hemisphere, depending upon the final Reynolds numbers and the acceleration of the inner sphere starting from subcritical flow, with the outer sphere at rest. One can see in the results presented ${ }^{14}$ that, in a certain range of final Reynolds numbers and rates of acceleration of the inner sphere, it is impossible to predict the flow state type. It appears that the selection of the number of Taylor vortices under these conditions was random.

Multiple flow states are possible also in a wide spherical gap, for example, at $\delta=1$, which is the configuration under study in this paper. With this aspect ratio, the stability limit was found to occur at $\operatorname{Re}_{\mathrm{c}}=460 \pm 2 .^{10,11}$ The case $\delta=1$ is especially interesting for investigation because calculated marginal stability curves, corresponding to the onset of linear instability for azimuthal waves with wave numbers $m=3$ and $m=4$ [Fig. 1(a)], cross near this gap size. ${ }^{12}$ It means that growth rates, $\lambda_{3}$ and $\lambda_{4}$ for linear modes $m=3$ and $m=4$, are close, but not equal to each other. Near the threshold of instability in the case of $\delta=1 \lambda_{3}<\lambda_{4},{ }^{11}$ suggesting that an azimuthal wave with $m=4$ would be preferred. Uncertainties are always present in experiments, but with small noise levels it is found that mode $m=4$ remains the preferred azimuthal wave, which is always established under conditions of quasi-static increases in Reynolds number. ${ }^{10,15}$ After the first instability, hysteresis between azimuthal wave numbers, $m$, was observed in experiments between either a quasi-static increase in $\operatorname{Re}$ (favouring $m=4$ ) or a decrease (favouring $m=3$ ). When the final Reynolds number, $\mathrm{Re}_{\text {fin }}$, was held constant and lies inside the observed region of hysteresis $(462<\operatorname{Re}<525)$, a loss of stability with increasing acceleration rate or decreasing initial Reynolds number results in a transition from $0 \rightarrow 3$ instead of $0 \rightarrow 4$, where "0" denotes the basic axisymmetric flow. ${ }^{15}$

The reasons for multiple flow states appearing under the action of different initial flow states and accelerations are well known for certain shallow and deep spherical layers. In a (Re, torque) bifurcation diagram for a thin layer direct numerical simulation (DNS) with $\delta=0.18,{ }^{16}$ the "preferred" 2-vortex flow is situated on the same branch as the initial 0vortex flow, while the 1-vortex flow position is on another non-intersecting branch. Imposing rapid accelerations in the forcing prevents switching between the branches, likely due to the long growth timescale of linear instability, and in this case a $0 \rightarrow 2$ transition occurs. ${ }^{16}$ When acceleration is slow and its duration is longer than the linear instability e-folding timescale, a $0 \rightarrow 1$ transition takes place. In a wide gap $(\delta$ $=1$ ) inner sphere acceleration results in a redistribution of the kinetic energy of the flow in space and time. ${ }^{17}$ First, the meridional circulation and azimuthal velocity maxima are shifted for a short time from the poles towards the equatorial plane. Second, the meridional component of kinetic energy, $\mathrm{E}_{\Psi}$, reaches its stationary value earlier than the azimuthal component, $\mathrm{E}_{\Phi}$, which implies an increase for a short time of the ratio $\mathrm{E}_{\Psi} / \mathrm{E}_{\Phi}$, compared with the steady boundary rotation. Under steady boundary conditions, $\mathrm{E}_{\Psi} / \mathrm{E}_{\Phi}$ is seen to increase with $\delta,{ }^{18,19}$ together with the wave number after the first instability decreases. So a short-term increase in the relative part of the meridional energy during acceleration of the boundaries can be considered as approximately equivalent to a short-term virtual increase in the relative gap size. This interpretation is consistent with the observed change in wave number from $0 \rightarrow 4$ to $0 \rightarrow 3$ under the action of an acceleration. It was shown in some range of initial values of $\mathrm{Re}_{\text {init }}$ at small acceleration rates that the preferred mode $(m=4)$ is the same as for a quasi-static increase in Re, while with larger acceleration rates the wavenumber selection became essentially random. ${ }^{15}$ It was suggested that random wave number selection, with acceleration values increasing within a restricted range of initial Re, can be explained by the influence of noise imposed in the form of deviations of the inner sphere rotational velocity from given values. ${ }^{17}$

In experiments and in nature, one generally cannot observe rotation of mechanical systems without experiencing at least some deviations from its average value. In nature, for example, there are well-known rotating neutron starspulsars, which emit radio pulses which are expected to be synchronized with the stellar rotation and hence highly regular and periodic (see, for example, Ref. 20). The physical origin of timing irregularities, which are commonly observed in the form of noisy, short-term fluctuations in the timing and amplitude of radio pulses ${ }^{20}$-remains unclear. Noise in these pulses is probably indicative of irregularities in the pulsar rotation rate. Short-term fluctuations are often interpreted as having been caused by a sudden increase in the angular velocity of the pulsars associated with "starquakes," and a detailed description of the analogy between pulsars and phenomena in SCF can be found in Ref. 21. The rotation of the Earth itself, as measured by space geodetic techniques, is also subject to fluctuations of order $0.1 \mathrm{~ms}$ on a variety of timescales, ranging from days to decades and longer. ${ }^{22}$ These fluctuations both in the length of the day and in the pole of rotation are generally attributed to changing motions in the Earth's atmosphere, oceans, and fluid core and their mutual angular momentum conserving interactions with the solid planet. So, there is a need to understand the influence of irregularities in background rotation on various flow systems.

For SCF, even if the influence of a constant acceleration rate is predictable, the response of the system for weak noise in the rotation rate up to now has been unclear. Of course, the effect of noise has been well studied for many classes of dynamical system. ${ }^{23}$ For example, additive noise can generate both a small shift in the critical values of a bifurcation parameter ${ }^{24}$ and instability in rotating shear flows. ${ }^{25}$ Noise injection can also lead to a frequency selection effect $^{26}$ and the creation of new states, ${ }^{27}$ since, in the presence of noise, a temporally coherent state may appear. ${ }^{28}$ But up to now only one experimental work is known where the influence of external noise on the first and higher instabilities in SCF was studied. ${ }^{29}$ It was shown in that study that, in the presence of small artificial disturbances, introduced into the flow by an 

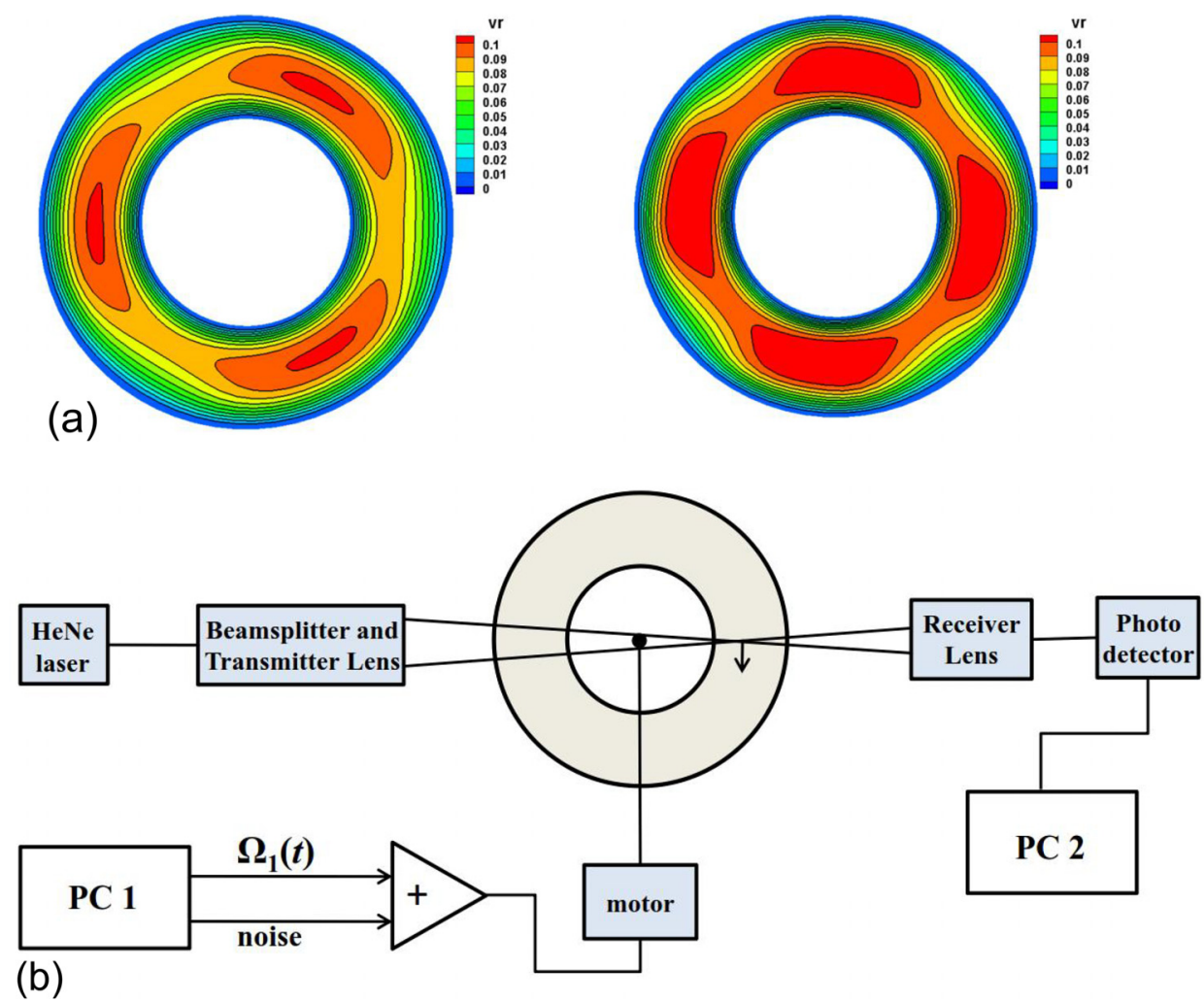

FIG. 1. (a) Contour lines of radial velocity component in equatorial plane for azimuthal waves with $m=3$ and $m=4$. The numerical simulations were done using the code, described in Ref. 34. (b) Scheme of the experimental setup. (c) Picture of the experimental setup.

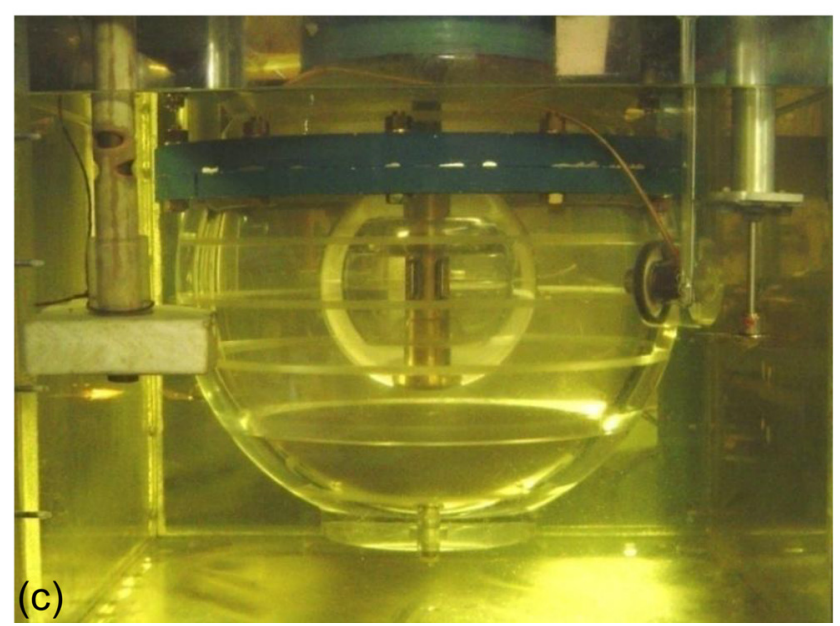

acoustic transducer, a shift in the critical Reynolds number of the first instability is small in comparison to its value.

The aim of this study is a detailed experimental investigation of the influence of weak noise perturbations on the flow state selection in SCF beyond the first instability. In particular, the case, where the final Reynolds number is reached following inner sphere acceleration from a subcritical flow to a supercritical one, is under consideration. Section II describes the experimental configuration and how the experiments were run, while Sec. III presents the main results. The main conclusions are summarised in Sec. IV, together with a discussion of their significance.

\section{RESEARCH METHODS AND MATERIALS}

A schematic diagram and a photograph of the experimental setup are shown in Figs. 1(b) and 1(c). A spherical layer was formed by two concentric and coaxial spheres, 0.075 and 0.150 $\mathrm{m}$ in radii, made from optically transparent acrylic resin. The aspect ratio of this layer was $\delta=1$. The spherical gap was filled with transparent silicon oil with a small addition of aluminium powder (less than $0.002 \%$ by volume) with the purpose to visualize the flow structure by the scattered light. The kinematic viscosity of the oil was about $\nu=5 \times 10^{-5} \mathrm{~m}^{2} / \mathrm{s}$ at $22^{\circ} \mathrm{C}$. To limit temperature deviations to within $\pm 0.05^{\circ} \mathrm{C}$, the spheres were placed in an optically transparent thermostat filled with circulating silicon oil. The inner sphere motion was controlled by a dedicated signal processor through a DC motor. Instantaneous values of the inner sphere angular velocity $\Omega$ were obtained by a standard method as the time derivative of a phase signal, determined from a rotation speed sensor (HEDS-5540) attached to the motor shaft. $\Omega$ could be kept constant or changed from initial to final $\Omega$ values linearly in time with constant angular acceleration $a=\mathrm{d} \Omega / \mathrm{dt}$. The control system maintained average 
magnitudes of $a$ and $\Omega$ with an accuracy of $\pm 0.05 \%$ (one can present $\Omega$ as a linear dependence on $\mathrm{t}$ by least squares fitting from which the error could be estimated). Some deflections from average values may be considered as a form of "noise" in the boundary conditions. As a quantification of the noise level, we use $\mathrm{N}=\frac{1}{\Omega} \sqrt{\frac{1}{K-1} \sum_{i=1}^{K}\left(\Omega\left(\mathrm{t}_{i}\right)-\bar{\Omega}\right)^{2}}$-representing the relative root mean square deviations of $\Omega(\mathrm{t})$ from its mean value, where $K$ is the length of the time series in numbers of timesteps. Because the end of damping and subsequent stage of growth for two competing linear modes occur after $\mathrm{Re}_{\text {fin }}$ is reached, ${ }^{15}$ we calculate value of $\mathrm{N}$ after the end of the acceleration phase. In our experiments, control settings were able to maintain $\Omega(\mathrm{t})$ to a precision $\mathrm{N}$ less than $0.95 \%$ from the prescribed values of $\Omega$, and this was effectively the minimum level of noise in these experiments. To investigate the influence of noise on flow state selection, however, we needed systematically to increase the noise amplitude. In our experiments, we used the maximum level of N, caused by imposed artificial disturbances, which was not more than $1.14 \%$, constrained by technological features of our setup. Artificially imposed small disturbances with a controlled amplitude were introduced to the $\Omega(\mathrm{t})$ signal to increase its rms variability. These disturbances were generated inside the control system. The aim of this system in the absence of additional noise is to produce at every time step a driving signal proportional to the difference between the actual and specified values of $\Omega$. When we preset a nonvanishing amplitude of the imposed noise, the desired value $\Omega(\mathrm{t})$ was changed by the addition of a uniformly distributed random value (which could be positive or negative), received from a random number generator. The amplitude of disturbances in each experiment was proportional to $\Omega(\mathrm{t})$ for a given $\mathrm{N}$ and therefore statistically stationary for time intervals with a fixed $\Omega(\mathrm{t})$. The actual $\Omega(\mathrm{t})$ signals were recorded to a PC for subsequent processing; $\Omega(\mathrm{t})$ without additional noise and with the maximum amplitude of additional noise are plotted for comparison in Fig. 2(a). The corresponding spectra, shown in Fig. 2(b), demonstrate near-

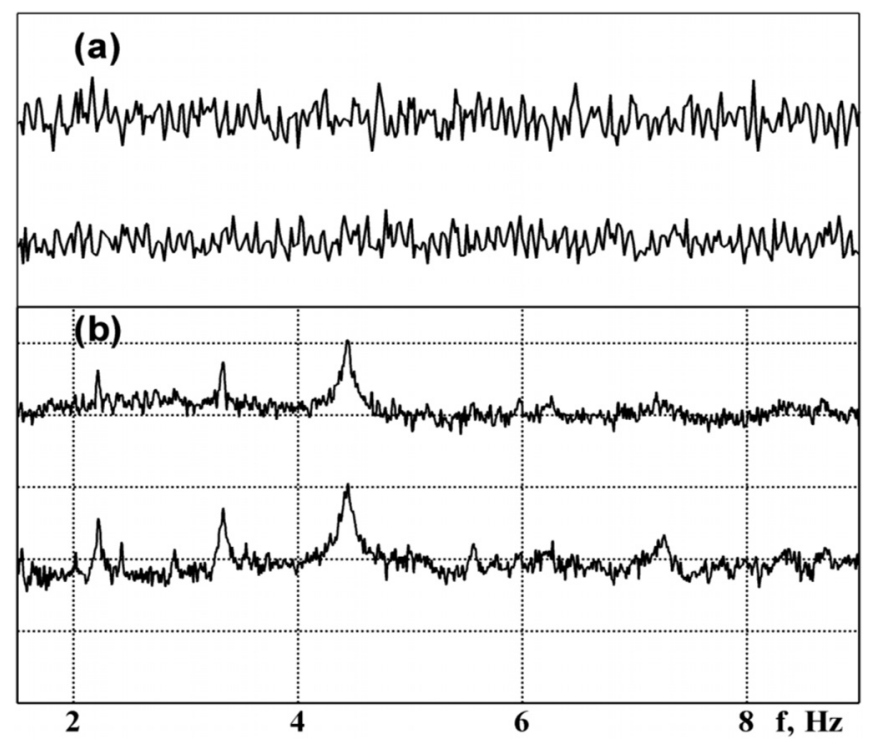

FIG. $2 . \Omega(\mathrm{t}) / 2 \pi$ signal with 20 s time length (a) and its amplitude spectral density (b): top [(a) and (b)] $\mathrm{N}=0.0112$, bottom [(a) and (b)] $\mathrm{N}=0.0095$. Both signals and spectra are separated by shifting along the vertical axes. uniform amplitudes in a broad band of frequencies (on both sides of a peak frequency at $4.5 \mathrm{~Hz}$ ) and this is an attribute of "white" noise. The difference between the spectra of $\Omega(t)$ signals with and without added noise of the largest amplitude seems to be imperceptible, because in both cases the origin of the noise is due to the action of the control system. So, the noise which we use in our experiments looks like white multiplicative noise, in accordance with its origin and kind of spectrum.

Fluctuations of the azimuthal velocity component were measured by laser Doppler anemometry using a forward scattering optical scheme, with the $25 \mathrm{~mW}$ HeNe laser and photo detector placed on different sides of the measurement volume. The high signal-to-noise ratio for our forward scattering optical arrangement allows us to obtain reliable measurements in both cases, either with one rotating sphere or with both spheres rotating independently. The LDV measurements and flow structure visualization were conducted simultaneously, similarly to the experiments described in Ref. 30. Measurements were carried out near the immobile outer sphere at a point located at a distance of $0.078 \mathrm{~m}$ from the equatorial plane and $0.105 \mathrm{~m}$ from the axis of rotation. The available range of velocities was from 0.005 to $1 \mathrm{~m} / \mathrm{s}$; the time series were recorded via an $\mathrm{AD}$-converter for further processing. The data acquisition sampling rate was $19.9 \mathrm{~Hz}$, while the frequency of the periodic flow after the first instability with the above-mentioned oil viscosity was $0.32 \mathrm{~Hz}$ $(m=3)$ or $0.43 \mathrm{~Hz}(m=4)$. The wave number of the secondary flow was obtained by counting the frequency of vortex transits.

The aim of each experiment was to determine the terminal wave number $m$ of the secondary flow at fixed specified values of acceleration rate and noise level. Each experiment was carried out in the following way: first of all, for a chosen noise amplitude, the stationary flow before the first instability with constant $\mathrm{Re}_{\text {init }}$ was maintained (for not less than $20 \mathrm{~min}$ ) and flow velocity measurements were begun. Then the acceleration of the inner sphere was started (with a linear in time variation of $\mathrm{Re}$ ) from $\mathrm{Re}_{\text {init }}$ up to $\mathrm{Re}_{\text {fin }}$, after which $\mathrm{Re}_{\text {fin }}$ was maintained constant for not less than $30 \mathrm{~min}$. Secondary flow developed during the time of acceleration and for some time after its end, resulting in one of the two possible modes. The established mode cannot be changed under current experiment conditions. Therefore, the next experiment in a sequence starts from the very beginning with newly set values of acceleration rate and noise level. Increases in Re also corresponded to increases in $\operatorname{rms}(\Omega(t))$. In order to eliminate the influence of a possible "memory effect" from previous flow states in the working fluid and in accordance with the methods used in Ref. 31, the inner sphere was held at rest for not less than 20 min between different runs. When needed, the experiments were repeated to ensure they led to reproducible results. In our experiments, flow velocity measurements were carried out with constant acceleration for the case when acceleration was begun from $\mathrm{Re}_{\text {init }}=100$ and was completed at $\mathrm{Re}_{\mathrm{fin}}=500$.

The flow state observed is determined by seven dimensional parameters: $\mathrm{r}_{1}, \mathrm{r}_{2}, \nu, \Omega_{\mathrm{i}}, \Omega_{\mathrm{f}}, \Delta \mathrm{t}$, and the noise amplitude. In addition to the relative gap size $\delta, \mathrm{Re}_{\text {init }}, \mathrm{Re}_{\text {fin }}$, and 
the relative noise amplitude $\mathrm{N}$, we use the dimensionless inner sphere acceleration

$$
\mathrm{dRe} / \mathrm{d} \tau=\left(\operatorname{Re}_{\text {fin }}-\operatorname{Re}_{\text {init }}\right) / \Delta \tau, \quad \Delta \tau=\Delta \mathrm{t} /\left(\mathrm{r}_{1}^{2} / \nu\right),
$$

where $r_{1}{ }^{2} / \nu$ is the radial diffusion time $e^{31}$ and $\Delta t$ is the time needed to achieve $\mathrm{Re}_{\text {fin }}$ from $\mathrm{Re}_{\text {init. }}$. In presenting the experimental results, we use the dimensionless time $\tau=\mathrm{t} /\left(\mathrm{r}_{1}{ }^{2} / \nu\right)$.

\section{RESULTS AND DISCUSSION}

First of all, we investigated whether small quasirandom disturbances in the rotation rate of the inner sphere affected the selection of the secondary flow mode in the absence of accelerations. In this sequence of experiments, Re was increased step by step, giving enough time for any transition from unsteady to steady-state flow to take place. In the absence of additional noise, the first transition for the gap aspect ratio $\delta=1$ was at $\operatorname{Re}_{\mathrm{c}}=460 \pm 2$ and was free of hysteresis, in contrast to the case for shallow layers, ${ }^{32}$ and occurs in accordance with linear instability theory. ${ }^{10,11}$ The solution of the linearized Navier-Stokes equations for this situation ${ }^{11}$ demonstrates exponential growth or decay of perturbations, and the real part of the exponent is the decrement/increment $\lambda$. In the range of $\mathrm{Re}$ from 460 up to 500, increments of growth for linear mode $\lambda_{4}(m=4)$ are greater than the corresponding increments for linear mode $\lambda_{3}(m=3) .{ }^{11,33}$ So, when Re increased quasi-statically (or step by step), secondary flow after the first instability with $m=4$ was established. In this experiment, it needed too much time (several hundred innersphere revolutions) to observe the threshold of the first instability with Re increasing. A more precise method to define $\mathrm{Re}_{\mathrm{c}}$ for a Hopf bifurcation, both in experiments and calculations, was to find the amplitudes of supercritical oscillations at different values of $\operatorname{Re}>\operatorname{Re}_{c}$. It was shown ${ }^{34}$ for a wide gap, $\delta=1.27$, for which these amplitudes, derived by DNS, are proportional to $\left(\mathrm{Re}-\mathrm{Re}_{\mathrm{c}}\right)^{1 / 2}$ near the critical point. This relation allows the estimation of the value $\operatorname{Re}_{\mathrm{c}}$ from a parabolic fit. In our case, with $\delta=1$, the amplitudes of secondary flow oscillations with $m=4$, received from LDV measurements, are plotted as a function of Re in Fig. 3. Even a parabolic approximation based on just three points shows that, both with and without additional noise, possible differences in $\mathrm{Re}_{\mathrm{c}}$ are negligible (for $\operatorname{Re}<466$ all points fit onto a solid curve, corresponding to the case without additional noise).

Now let us consider the influence on the wave number selection of imposing additional noise in the presence of acceleration. More interesting is the case with $m=3$ in the secondary flow in the absence of additional noise. When transition from $\mathrm{Re}_{\text {init }}$ to $\mathrm{Re}_{\text {fin }}$ through $\mathrm{Re}_{\mathrm{c}}$ occurs with acceleration, the relation between the increments $\lambda_{3}$ and $\lambda_{4}$ is found to change. Thus, results of numerical calculations show that, with $\mathrm{Re}_{\text {init }}$ decreasing ${ }^{33}$ or with acceleration increasing, ${ }^{17}$ short-term conditions arise which lead to $\lambda_{4}$ $<\lambda_{3}$. This is a reason for selection of secondary flow with $m=3$, because the flow keeps a memory of its history. We suggest that the above-mentioned short-term conditions may

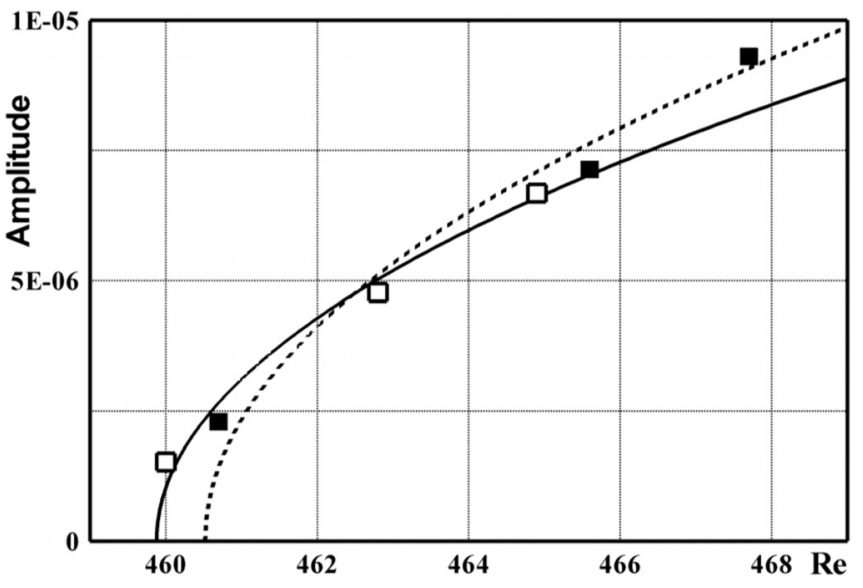

FIG. 3. Amplitudes of secondary flow with $m=4$ : open symbols (solid line as approximation) $-\mathrm{N}=0.0095$ and dark symbols (dashed line) $-\mathrm{N}=0.01$.

be "forgotten" in the presence of additional noise, and a transition from $0 \rightarrow 4$ may be possible under the action of additional noise instead of transition $0 \rightarrow 3$. Our suggestion is consistent with a well known result: in the Lorenz model with random forcing, it was shown that noise causes an additional loss of memory. ${ }^{35}$ Wave numbers $m$, obtained from visualization observations at $\mathrm{Re}_{\text {init }}=100$ and two values of acceleration, are presented in Fig. 4 as a function of $\mathrm{N}$. Every point in Fig. 4 represents the result of determining $m$ obtained in corresponding experiment. It is clearly seen that, for each acceleration value, an increase in $\mathrm{N}$ leads to a change of preferred mode, namely, from a transition $0 \rightarrow 3$ to transition $0 \rightarrow 4$. Moreover, the threshold level of noise required for the change of $m$ depends on the acceleration rate.

The data from experiments, such as presented in Fig. 4 and also obtained by means of visualization, are summarized in Fig. 5. Every point in Fig. 5 is the result of several independently executed experiments (as detailed in Sec. II) and denotes the threshold value of $\mathrm{N}$ at which a change from $m=3$ to $m=4$ was found to occur with $\mathrm{N}$ increasing at a constant value of $\mathrm{dRe} / \mathrm{d} \tau$. A range of $200-2000$ for $\mathrm{dRe} / \mathrm{d} \tau$ was found to be sufficient to investigate the influence of a given

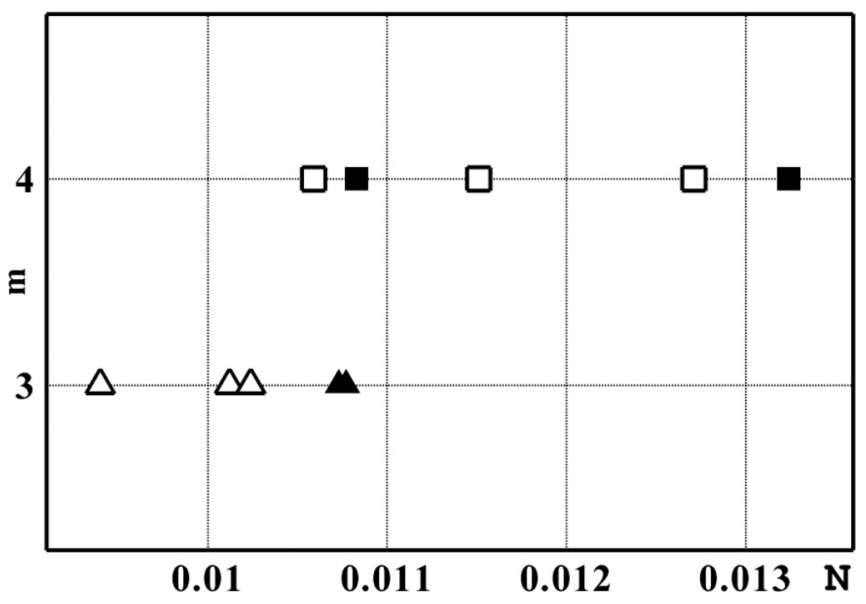

FIG. 4. Wave number $m$ depending on noise level $\mathrm{N}$ in the case $\mathrm{Re}_{\text {init }}=100$. Triangular symbols correspond to $m=3$ and square- $m=4$. Open symbols- $\mathrm{dRe} / \mathrm{d} \tau=636$ and dark symbols- $\mathrm{dRe} / \mathrm{d} \tau=795$. 


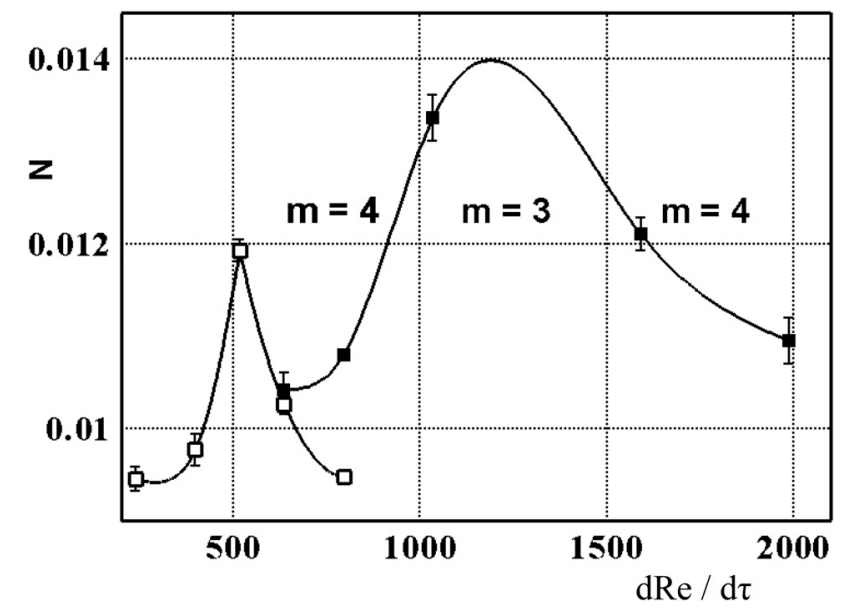

FIG. 5. Thresholds of wave number alterations: symbols-experiment results, lines-approximation, open symbols correspond to $\mathrm{Re}_{\text {init }}=0$, and closed symbols correspond to $\mathrm{Re}_{\text {init }}=100$. The area under every line corresponds to the case when $0 \rightarrow 3$ transition occurs, the area above-to the case of a $0 \rightarrow 4$ transition. Sizes of the error bars correspond to the difference between neighboring $\mathrm{N}$ values with different $m$ and error bars range from $10^{-4}$ to $5 \cdot 10^{-4}$.

level of noise on the wave number selection. Solid lines represent cubic spline approximations to the experimental points. Above each approximation line in Fig. 5, the wave number is $m=4$, while under the line, the flow is dominated by $m=3$. Each approximation line rises to a local maximum and the greater the initial $\mathrm{Re}$, the larger the acceleration corresponding to this maximum. Note that although the approximation applied in our study cannot pinpoint the exact position of the maximum, it reveals the general qualitative behavior. Two consequences may be deduced from these results. First, with increasing $\mathrm{dRe} / \mathrm{d} \tau$ at a large enough constant $\mathrm{N}$ level (for example, $0.0115<\mathrm{N}<0.013$ at $\mathrm{Re}_{\text {init }}=100$ shown as closed symbols in Fig. 5), we observed two changes of $m$. At small acceleration rates $(\mathrm{dRe} / \mathrm{d} \tau<1000)$ we observed a change of $m$ from 4 to 3 , while at larger rates $(\mathrm{dRe} / \mathrm{d} \tau>1500)$ we observed a transition $0 \rightarrow 4$ instead of $0 \rightarrow 3$. Second, threshold curves were found to depend on $\mathrm{Re}_{\text {init }}$ : with increasing $\mathrm{Re}_{\text {init }}$, the threshold values of $\mathrm{dRe} / \mathrm{d} \tau$ became larger. It is interesting to note that these consequences from experimental data are in agreement with numerical results, obtained earlier with fixed $\mathrm{Re}_{\mathrm{fin}}{ }^{17}$ where, in the $\mathrm{Re}_{\text {init }}-\mathrm{dRe} / \mathrm{d} \tau$ diagram, the same sequence of changes to the dominant wave number 4 $\rightarrow 3 \rightarrow 4$ was observed with $\mathrm{dRe} / \mathrm{d} \tau$ increasing. It is possible that a velocity field divergence greater than $10^{-6}$ (due to the controlled inaccuracy of the numerical calculation algorithm) in the previously mentioned numerical results may be considered as additional noise. The dependence of thresholds on acceleration rate for the case $\mathrm{Re}_{\text {init }}=100$ seems to be asymmetric about the maximum (Fig. 5). It means that, at a constant and sufficiently small noise level $\mathrm{N}<0.011$ and $\mathrm{dRe} / \mathrm{d} \tau$ no larger than 2000 , there is only one change of dominant wavenumber from $m=4$ to $m=3$ (or one needs to increase $\mathrm{dRe} / \mathrm{d} \tau$ still further to find the other alteration of wavenumber). Just the same results - a $0 \rightarrow 3$ transition instead of 0 $\rightarrow 4$ with $\mathrm{dRe} / \mathrm{d} \tau$ increasing-were observed earlier in experiments without additional noise, ${ }^{15}$ where control settings provided a smaller minimal $\mathrm{N}$ in comparison with the same quantity used in this work.

The results presented in this study therefore demonstrate three possible scenarios for wave number selection as a function of noise amplitude. The first scenario is realized for very small noise amplitudes, and the wave number $m=3$ appears to be independent of acceleration rate. This first scenario was observed in experiments and calculations, ${ }^{15,17}$ when acceleration was begun from the state of rest or with very slow flow $\left(\mathrm{Re}_{\text {init }}=0-10\right)$. In the second scenario, for bigger noise amplitudes, one (from $0 \rightarrow 4$ to $0 \rightarrow 3$ ) or two (from $0 \rightarrow 4$ to $0 \rightarrow 3$ at smaller values of $\mathrm{dRe} / \mathrm{d} \tau$ and from $0 \rightarrow 3$ to 0 $\rightarrow 4$ at larger values) transitions were found to occur. The second scenario was revealed with $100<\mathrm{Re}_{\text {init }}<450$ as the result of direct numerical simulations. ${ }^{17}$ The third scenario, not observed before, arises with large enough noise amplitudes and resulted in the wave number $m=4$ appearing independently of the acceleration rate. The first and third scenarios are the simple marginal cases. In the first scenario, a significant acceleration influence allows one to ignore the presence of noise; cases where noise takes no action are well known (see, e.g., Ref. 36) In the third scenario, a large noise level allows neglect of the influence of prehistory, induced by acceleration, but this does not contradict the results obtained in Ref. 35. The most complex is the second scenario with extreme noise-to-acceleration ratio.

At the left hand ascending part of each approximated regime boundary in Fig. 5, increasing $\mathrm{N}$ leads to an increased value of $\mathrm{dRe} / \mathrm{d} \tau$ necessary to bring about the change from 0 $\rightarrow 4$ to $0 \rightarrow 3$, while at the descending side of the regime boundary, larger values of $\mathrm{dRe} / \mathrm{d} \tau$ lead to a lower threshold value of $\mathrm{N}$. At the ascending part, increasing $\mathrm{dRe} / \mathrm{d} \tau$ leads to an amplification of the factors favouring a change of preferred wave number from 4 to 3 , as discussed in the introduction. The descending part may probably be explained as follows. As was mentioned above, the flow is unsteady both during acceleration and for some time after its completion. The quicker the acceleration is, the larger is the full unsteady time, ${ }^{17}$ and the less noise amplitude is necessary for a transition between modes. So, the wave number selection under action of both acceleration and noise is a complicated problem, and below we only consider part of it, namely, the influence of noise at a fixed acceleration value.

To elucidate the role of noise in wave number selection, the behaviour of the amplitudes of competing modes $\left[A_{3}(\tau)\right.$ for $m=3$ and $A_{4}(\tau)$ for $m=4$, respectively] during their stages of damping and growth was examined from time series of measured flow velocity $u(t)$. A detailed description of the behaviour of axisymmetric modes under acceleration was carried out in a series of numerical experiments ${ }^{37}$ in cylindrical Taylor-Couette flow. These authors have shown that, for all linear modes, a short period of decay occurs before exponential growth begins. Even if the preferred mode is initially damped faster than some of the others, it begins to grow before the other modes and its linear growth rate is larger as well. The same kind of behaviour of the amplitudes of the non-axisymmetric linear modes-in which a preferred mode begins to grow earlier than the others- 
was observed in experiments with an acceleration of the inner sphere in Ref. 15.

In our experiments, to avoid operating with large noise levels, measurements were carried out at an acceleration rate $\mathrm{dRe} / \mathrm{d} \tau=398$ (see Fig. 5). Two methods were used to define the evolution of modal amplitudes in time. In the first, the same as in Ref. 15, amplitudes were represented as the amplitudes of corresponding components of the frequency spectrum obtained via a Fast Fourier Transform (FFT), applied to a constant-width window moving along the time axis. In the second method, analogous to Ref. 38, we calculated $A_{3}(\tau)$ and $A_{4}(\tau)$ using the Hilbert Transform (HT). In the latter method, it is necessary to extract components $\mathrm{u}_{3}(\mathrm{t})$ and $\mathrm{u}_{4}(\mathrm{t})$ first, corresponding to the modes $m=3$ and $m=4$, from the whole original signal $u(t)$. Because of the absence of other peaks, except for $f_{3}$ and $f_{4}$, in the spectrum of $u(t)$ it is sufficient to use the band-pass filtering with a rectangular window based on a FFT: $\tilde{u}(f)=F F T(u(t)), u_{i}(t)=F F T^{-1}\left(\tilde{u}\left(f_{i} \pm d f\right)\right)(i=3,4)$, where $f_{i}$ are known: $f_{3}=0.32 \mathrm{~Hz}$ and $f_{4}=0.43 \mathrm{~Hz}$ (Sec. II). We tested different $d f$ values and selected $d f=0.05 \mathrm{~Hz}$ for both frequencies. We therefore use the case when rectangular windows for neighboring frequencies $f_{3}$ and $f_{4}$ do not intersect with each other; smaller values of $d f$ lead to non-uniform in time amplitude oscillations for the damped mode, whereas larger values do not improve the filtering.

For each mode, we define amplitude as the modulus of the analytical signal

$$
A_{i}(t)=\left|u_{i}(t)+\mathrm{iHT}\left(u_{i}(t)\right)\right| .
$$

The main difference between the two approaches is that in using a FFT to obtain the amplitude over a specific interval in time, we use only part of the time series - a window, and the result does not depend on the points outside of this window. In contrast, using the HT method we obtain the time dependence of each amplitude using all the points in the time series.

The resulting amplitudes of linear modes $A_{3}(\tau)$ and $A_{4}(\tau)$, calculated by the first method (via an FFT in consecutive windows), are shown in Fig. 6 as a function of non-dimensional

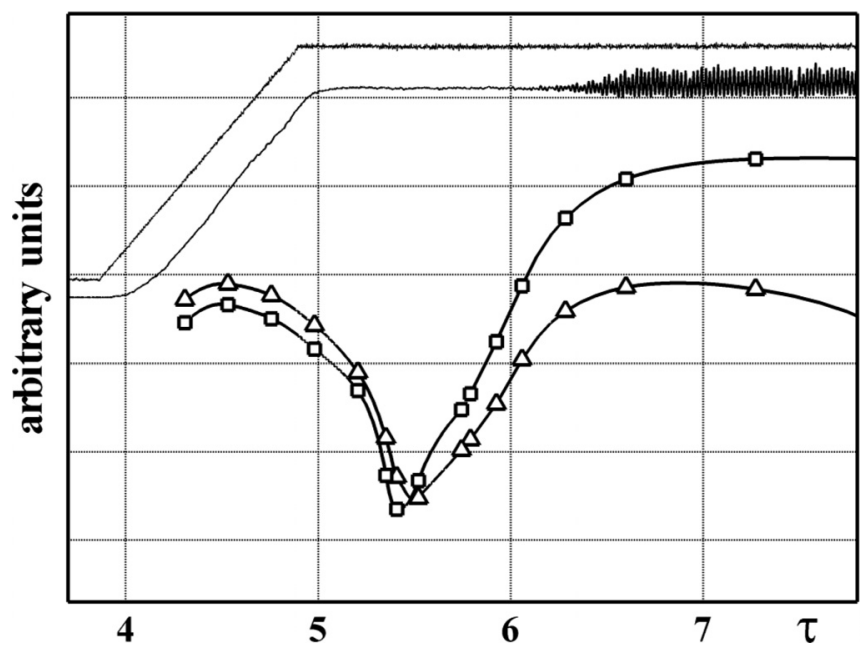

FIG. 6. Evolution in time: $\Omega(\mathrm{t}) / 2 \pi$-top line without symbols, $u(t)$-bottom line without symbols, $A_{4}$-line with square symbols, $A_{3}$-line with triangular symbols every 5 th symbol is plotted, and $A_{4}$ and $A_{3}$ are in log scale. time $\tau$. Data correspond to the threshold for transition (from 0 $\rightarrow 3$ to $0 \rightarrow 4$ ) with relative noise level $N=0.0103$. By this example, it is possible to view the typical evolution of the main linear modes. During the acceleration time $(\tau=4-6)$, amplitudes are observed to rise to local maxima at around $\tau=4.6$, in agreement with the results of numerical simulations under the action of an acceleration, ${ }^{17}$ where $A_{3}$ became larger than $A_{4}$. This is followed by a short period when both modes decay before a transition from damping to growth of both linear modes and the next significant evolution in time occurs at constant angular velocity after the end of the initial acceleration. This period of secondary growth also includes a linear stage. The growth rate of the preferred mode is observed to be not less than the one for the other mode. The preferred mode goes on to achieve saturation, after which its amplitude becomes approximately constant, while the other mode begins its terminal decay after going through a secondary maximum. This type of linear mode behaviour is common for all the imposed noise levels used in our experiments and does not appear to depend on the data processing method. Such behaviour of these linear modes in the presence of noise is in agreement with results obtained earlier from numerical simulations in cylindrical Taylor-Couette flow ${ }^{37}$ and other experiments in SCF. ${ }^{15}$

Let us consider now how noise activity changes the interactions of the linear modes. For this, it is necessary to examine quantitatively the time evolution for the amplitudes of the preferred $\left(A_{P}\right)$ and damped $\left(A_{D}\right)$ modes and the magnitudes of their growth rates. The following parameters, varying depending on noise level, are presented in Fig. 7:

- $T_{1}=t\left(A_{D \min }\right)-t\left(A_{P \min }\right)$ is the time shift between points $A_{P \min }$ and $A_{D \text { min }}$;

- $T_{2}=t\left(A_{D \max }\right)-t\left(A_{P \min }\right)$ is the time shift between the beginning of preferred mode growth $A_{P \min }$ and the maximum amplitude of damped mode $A_{D \max }$.

The dependencies of $T_{1}$ and $T_{2}$ on noise level $\mathrm{N}$ are presented in Fig. 8. First of all, a good agreement is observed between results obtained by means of both the first (FFT) and second (HT) data processing methods; henceforth we

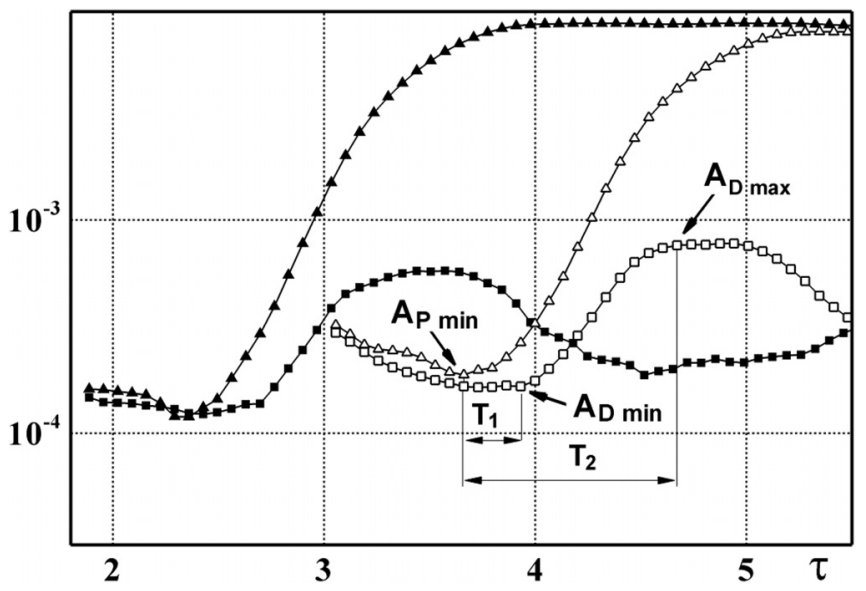

FIG. 7. $A_{3}(\tau)$ (line with triangular symbols) and $A_{4}(\tau)$ (line with square symbols), calculated by HT; without additional noise (open symbols) and $\mathrm{N}=0.01$ (closed symbols); time shift between different $\mathrm{N}$ cases is arbitrary. 


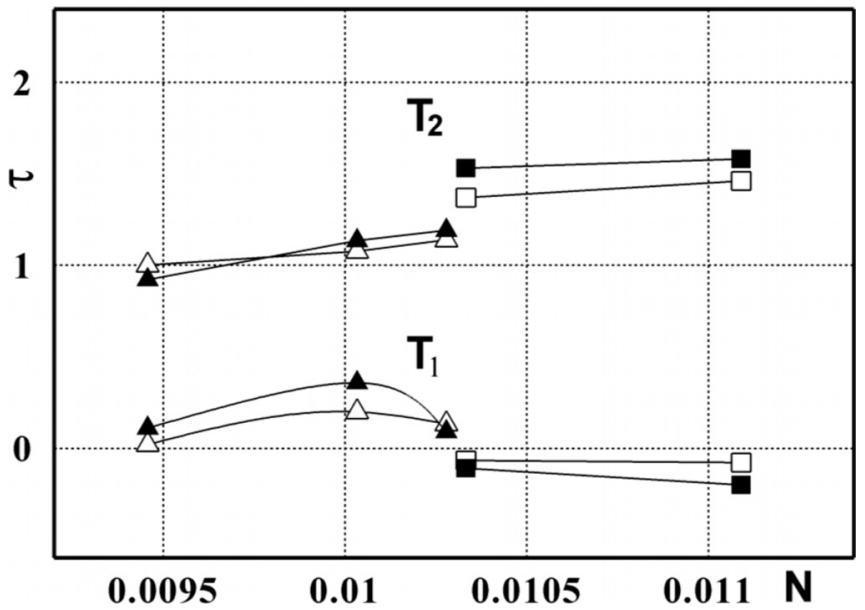

FIG. 8. Time intervals $T_{1}$ (bottom) and $T_{2}$ (top), open symbols-measured from FFT calculations, closed symbols-measured from HT calculations, depending on noise level N. $m=3$-triangle symbols and $m=4$-square symbols.

present the data from the HT method only. The complete time interval of linear mode interaction is designated as $T_{2}$, and increases with noise level growth for $\mathrm{N}<0.0103$. However, it then displays a small jump with $\mathrm{N}$ around where there is a change in wave number $m$ from 3 to 4 in the secondary flow. Similar jumps in the magnitudes of the inferred increments of linear growth, $\lambda_{3}$ and $\lambda_{4}$, were observed near the noise level that corresponds to a change in $m$ (Fig. 9). The increment of linear growth, $\lambda$, was defined as follows. Every curve $\mathrm{A}(\tau)$, presented using a logarithmic amplitude scale (e.g., Fig. 7), has a linear part for an interval after reaching $\mathrm{A}_{\text {min }} ; \lambda$ is defined as the slope of this part, thus representing an estimate of the local growth rate. The smallest difference between $\lambda_{3}$ and $\lambda_{4}$ (Fig. 9) was found at the highest noise amplitude (of course, eliminating the area near the alteration point, where the duration of the linear stage of growth is minimal, relative to the other cases).

The overall picture of noise action is as follows: in the presence of the noise the timescale of linear mode interaction, $T_{2}$, increases. The larger the noise level the stronger the damping of both linear modes and hence the smaller the differences

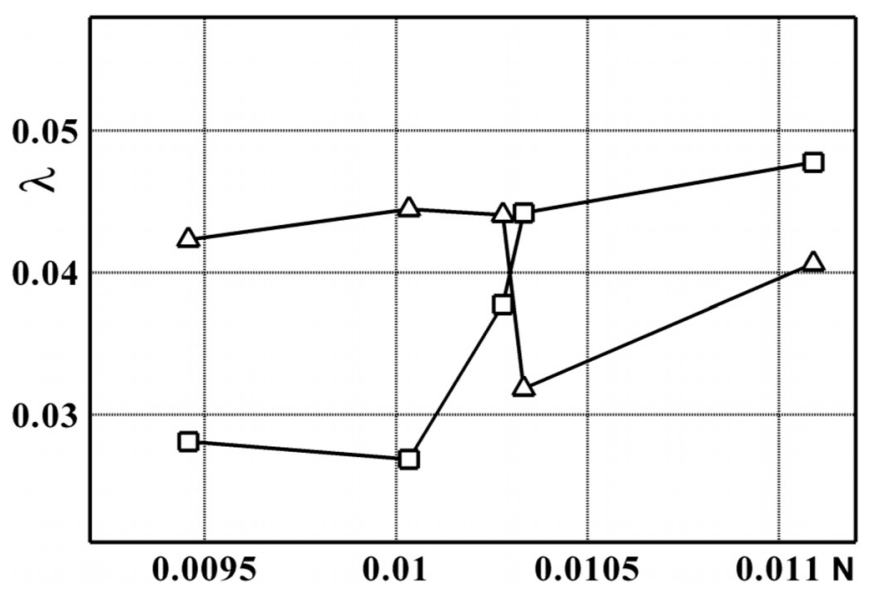

FIG. 9. Increments of growth for $m=3$ (triangular symbols) and $m=4$ (square symbols) depending on noise level N. in parameters associated with linear mode interactions-i.e., the increments of growth and maximal amplitudes.

\section{CONCLUSIONS}

In a series of spherical Couette flow experiments, we have considered how the selection of one from two possible wave numbers in the onset of azimuthal waves following the first instability depends on noise amplitude in the presence of an acceleration of the inner sphere. We have measured velocity fluctuations in the case when $\mathrm{Re}_{\text {init }}=100, \mathrm{Re}_{\mathrm{fin}}$ $=500$, and $\mathrm{dRe} / \mathrm{d} \tau=398$ and only the noise amplitude was varied from experiment to experiment (in every experiment the mean noise amplitude was kept constant). The following conclusions have been obtained. With noise amplitude increasing, an alteration from a $0 \rightarrow 3$ transition to a $0 \rightarrow 4$ transition was observed. It was shown that the action of the imposed noise leads to energy transfer from the initially preferred to the corresponding damped mode, increasing both its maximum amplitude and increment of growth. The imposed noise in the forcing also increases the total timescale of linear mode interactions, ranging from the beginning of ultimate growth of the preferred mode to the time when the damping mode reaches its maximum amplitude.

The sequence of data was obtained by means of flow visualization at $\mathrm{Re}_{\text {init }}=0$ and 100 . It was revealed that several scenarios for wave number transitions as a function of noise amplitude can be observed with increasing acceleration rate. At relatively "small" and "large" noise amplitudes, the favoured wave numbers may remain constant $-m=3$ in the first case and $m=4$ in the second case at any acceleration rates. The values of "small" and "large" noise amplitudes were found to be dependent on initial Re number, corresponding to the beginning of the acceleration phase. At intermediate noise levels, one change in wave number (from transition $0 \rightarrow 4$ to $0 \rightarrow 3$ ) or two successive changes (from transition $0 \rightarrow 4$ to $0 \rightarrow 3$ and then from $0 \rightarrow 3$ to $0 \rightarrow 4$ ) were observed with acceleration rate increasing. The greater the initial value of $\mathrm{Re}$ the larger the acceleration rates and noise amplitudes that corresponded to $m$ alterations. Further experimental and numerical studies are needed to understand the reasons behind these effects.

We suppose that investigations of noise action on the flow state selection are very important not only for their application to astrophysical objects, such as pulsars, but also to the selection of circulation regimes in the atmosphere. In Ref. 39, for example, a suggestion was made that for a better understanding of the selection of such atmospheric circulation regimes, such as those involving interactions of Rossby waves and transitions between them, it is necessary to consider state dependent noise. Theoretical models have been used to estimate the influence of noise action in condensed matter phase transitions, for which the noise intensity was shown to be a control parameter: ${ }^{40}$ a low noise intensity induces a phase transition towards an ordered state, whereas strong noise plays a destructive role. Such behavior can be expected to occur also in pattern formation in fluid flow. In our experiments, the role of weak and strong noise is not far from the situation described above if we consider the transition to the mode 
$m=3$ under the action of acceleration as an "ordered state": the strong noise destroys the results of an acceleration in the boundary conditions and the persistence of the preferred wave number $m=4$ is observed. A similar behavior of model systems, including the case of an imperfect pitchfork bifurcation, was observed in Ref. 41, where white and multiplicative noise were shown to suppress solutions that exist in the absence of noise. Such experimental results on the effects of noisy forcing are not restricted to the case of SCF. Effects on wave number transitions with increasing acceleration for constant noise intensity, and with noise intensity increasing at constant acceleration, as obtained in our work, were also observed in experiments ${ }^{27}$ on surface water waves, generated under the action of sinusoidal forcing. We suspect that other examples of similar effects of imposing noise perturbations on wave number selection from multiple stable solutions to nonlinear dynamical systems in the presence of forcing may also be observed for other instabilities.

\section{ACKNOWLEDGMENTS}

The authors sincerely thank the reviewers, Professor Jürgen Kurths, and Dr. Andrew Ball for their valuable advice and useful comments, which helped us to improve the manuscript. This work was supported by the Russian Foundation for Basic Research, Project Nos. 16-05-00004 and 18-08-00074. M.G. was supported, in part, by the ERC Advanced Grant No. 320773 (SAEMPL). The research at the Ural Federal University was supported by the Government of the Russian Federation (Act 211, Agreement 02.A03.21.0006).

${ }^{1}$ D. Coles, "Transition in circular Couette flow," J. Fluid Mech. 21, 385-425 (1965).

${ }^{2}$ T. B. Benjamin and T. Mullin, "Anomalous modes in the Taylor experiment,” Proc. R. Soc. London, A 377, 221-249 (1981).

${ }^{3}$ W. G. Frueh and P. L. Read, "Experiments on a barotropic rotating shear layer. Part 1. Instability and steady vortices," J. Fluid Mech. 383, 143-173 (1999).

${ }^{4}$ F. Feudel, L. S. Tuckerman, M. Zaks, and R. Hollerbach, "Hysteresis of dynamos in rotating spherical shell convection," Phys. Rev. Fluids 2, 053902 (2017)

${ }^{5}$ P. L. Read, E. P. Perez, I. M. Moroz, and R. M. B. Young, "General circulation of planetary atmospheres: Insights from rotating annulus and related experiments," in Modeling Atmospheric and Oceanic Flows: Insights From Laboratory Experiments and Numerical Simulations, edited by T. Von Larcher and P. D. Williams (American Geophysical Union and John Wiley, Washington DC and Hoboken, New Jersey, 2015).

${ }^{6} \mathrm{G}$. Khlebuytin, "Stability of fluid motion between a rotating and a stationary concentric sphere," Fluid Dyn. 3, 31-32 (1968).

${ }^{7}$ A. Child, R. Hollerbach, and E. Kersale, "Axisymmetrical pulse train solution in narrow-gap spherical Couette flow," Physica D 348, 54-59 (2017).

${ }^{8} \mathrm{~K}$. Bühler, "Symmetric and asymmetric Taylor vortex flow in spherical gaps," Acta Mech. 81, 3-38 (1990).

${ }^{9} \mathrm{C}$. Mamun and L. Tuckerman, "Asymmetry and Hopf bifurcation in spherical Couette flow," Phys. Fluids 7, 80-91 (1995).

${ }^{10}$ Y. Belyaev, A. Monakhov, and I. Yavorskaya, "Stability of spherical Couette flow in thick layers when the inner sphere revolves," Fluid Dyn. 13, 162-168 (1978).

${ }^{11}$ G. Dumus, "Study of spherical Couette flow via 3-D spectral simulation: Large and narrow-gap flows and their transitions," Ph.D. thesis (California Institute of Technology, 1991).

${ }^{12}$ R. Hollerbach, M. Junk, and C. Egbers, "Non-axisymmetric instabilities in basic-state spherical Couette flow," Fluid Dyn. Res. 38, 257-273 (2006).
${ }^{13}$ O. Sawatzki and J. Zierep, "Das stromfeld im spalt zwischen zwei konzentrishen kugelflächen, von denen die innere rotiert," Acta Mech. 9, 13-35 (1970).

${ }^{14} \mathrm{M}$. Wimmer, "Experiments on a viscous fluid flow between concentric rotating spheres," J. Fluid Mech. 78, 317-335 (1976).

${ }^{15} \mathrm{D}$. Zhilenko and O. Krivonosova, "Choice of flow regime in a wide spherical layer under the action of acceleration of the inner sphere," Dokl. Phys. 52(8), 453-457 (2007).

${ }^{16} \mathrm{P}$. Marcus and L. Tuckerman, "Simulation of flow between concentric rotating spheres, Part 2, transitions," J. Fluid Mech. 185, 31-65 (1987).

${ }^{17}$ D. Zhilenko and O. Krivonosova, "Direct calculation of transition to one of two possible secondary flows in a wide spherical layer," Fluid Dyn. 46, 363-374 (2011).

${ }^{18}$ I. Yavorskaya, Y. Belyaev, and A. Monakhov, "Hydrodynamical stability in rotating spherical layers-applications to dynamics of planetary-atmospheres," Acta Astronaut. 13, 433-440 (1986).

${ }^{19} \mathrm{C}$. Egbers and H. Rath, "The existence of Taylor vortices and wide-gap instabilities in spherical Couette flow," Acta Mech. 111, 125-140 (1995).

${ }^{20}$ Y. Wang, "Noise in pulsar timing arrays," J. Phys.: Conf. Ser. 610, 012019 (2015).

${ }^{21}$ C. Peralta, A. Melatos, M. Giacobello, and A. Ooi, "Superfluid spherical Couette flow," J. Fluid Mech. 609, 221-274 (2008).

${ }^{22}$ R. Hide and J. O. Dickey, "Earth's variable rotation," Science 253(5020), 629-637 (1991).

${ }^{23} \mathrm{~W}$. Horsthemke and R. Lefever, Noise-Induced Transitions (Springer, Berlin, 1984).

${ }^{24}$ A. Juel, A. Darbyshire, and T. Mullin, "The effect of noise on pitchfork and Hopf bifurcations," Proc. R. Soc. London A 453, 2627-2647 (1997).

${ }^{25}$ B. Mukhopadhyay and A. Chattopadhyay, "Stochastically driven instability in rotating shear flows," J. Phys. A: Math. Theor. 46, 035501 (2013).

${ }^{26}$ M. Proctor, S. M. Tobias, and E. Knobloch, "Noise-sustained structures due to convective instability in finite domains," Physica D 145, 191-206 (2000).

${ }^{27}$ R. Berthet, A. Petrossian, S. Residori, B. Roman, and S. Fauve, "Effect of multiplicative noise on parametric instabilities," Physica D 174, 84-99 (2003).

${ }^{28} \mathrm{X}$. Yang, D. Senthilkumar, and J. Kurths, "Impact of connection delays on noise-induced spatiotemporal patterns in neuronal networks," Chaos 22 , 043150 (2012).

${ }^{29}$ K. Nakabayashi, W. Sha, and Y. Tsuchida, "Relaminarization phenomena and external-disturbances effects in spherical Couette flow," J. Fluid Mech. 534, 327-350 (2005).

${ }^{30} \mathrm{P}$. Wulf, C. Egbers, and H. Rath, "Routes to chaos in wide-gap spherical Couette flow," Phys. Fluids 11(6), 1359-1372 (1999).

${ }^{31}$ Q. Xiao, T. Lim, and Y. Chew, "Effect of acceleration on the wavy Taylor vortex flow," Exp. Fluids 32, 639-644 (2002).

${ }^{32}$ G. Schrauf, "The first instability in spherical Taylor-Couette flow," J. Fluid Mech. 166, 287-303 (1986).

${ }^{33}$ D. Zhilenko and O. Krivonosova, "Competition of linear modes in a spherical Couette flow after sudden increase of rotational velocity of the inner sphere," Dokl. Phys. 54(11), 520-523 (2009).

${ }^{34}$ N. Nikitin, "Finite-difference method for incompressible Navier-Stokes equations in arbitrary orthogonal curvilinear coordinates," J. Comput. Phys. 217, 759-781 (2006).

${ }^{35}$ A. Zippelius and M. Lücke, "The effect of external noise in the Lorenz model of the Bénard problem,” J. Stat. Phys. 24(2), 345-358 (1981).

${ }^{36}$ V. Chandrasekar, J. Sheeba, B. Subash, M. Lakshmanan, and J. Kurths, "Adaptive coupling induced multi-stable states in complex networks," Physica D 267, 36-48 (2014).

${ }^{37}$ J. Rigopoulos, J. Sheridan, and M. Thompson, "State selection in Taylorvortex flow reached with an accelerated inner cylinder," J. Fluid Mech. 489, 79-99 (2003).

${ }^{38}$ L. Pastur, F. Lusseyran, T. Faure, Y. Fraigneau, R. Pethieu, and P. Debesse, "Quantifying the nonlinear mode competition in the flow over an open cavity at medium Reynolds number," Exp. Fluids 44, 597-608 (2008).

${ }^{39}$ P. Sura, M. Newman, C. Penland, and P. Sardeshmukh, "Multiplicative noise and non-Gaussianity: A paradigm for atmospheric regimes?," J. Atmos. Sci. 62, 1391-1409 (2005).

${ }^{40}$ J. Garcia-Ojalvo, J. M. R. Parrondo, J. M. Sancho, and C. Van den Broeck, "Reentrant transition induced by multiplicative noise in the timedependent Ginzburg-Landau model,” Phys. Rev. E 54, 6918-6921 (1996).

${ }^{41} \mathrm{~F}$. Petrelis and S. Aumaitre, "Modification of instability process by multiplicative noises," Eur. Phys. J. B 51, 357-362 (2006). 